\title{
SurgeryCuts: Embedding Additional Information in Maps without Occluding Features
}

\author{
M. Angelini ${ }^{1}$ and Juri Buchmüller ${ }^{2}$ and Daniel A. Keim ${ }^{2}$ and Philipp Meschenmoser $^{2}$ and G. Santucci ${ }^{1}$ \\ ${ }^{1}$ Sapienza University of Rome, Rome, Italy ${ }^{2}$ University of Konstanz, Konstanz, Germany
}

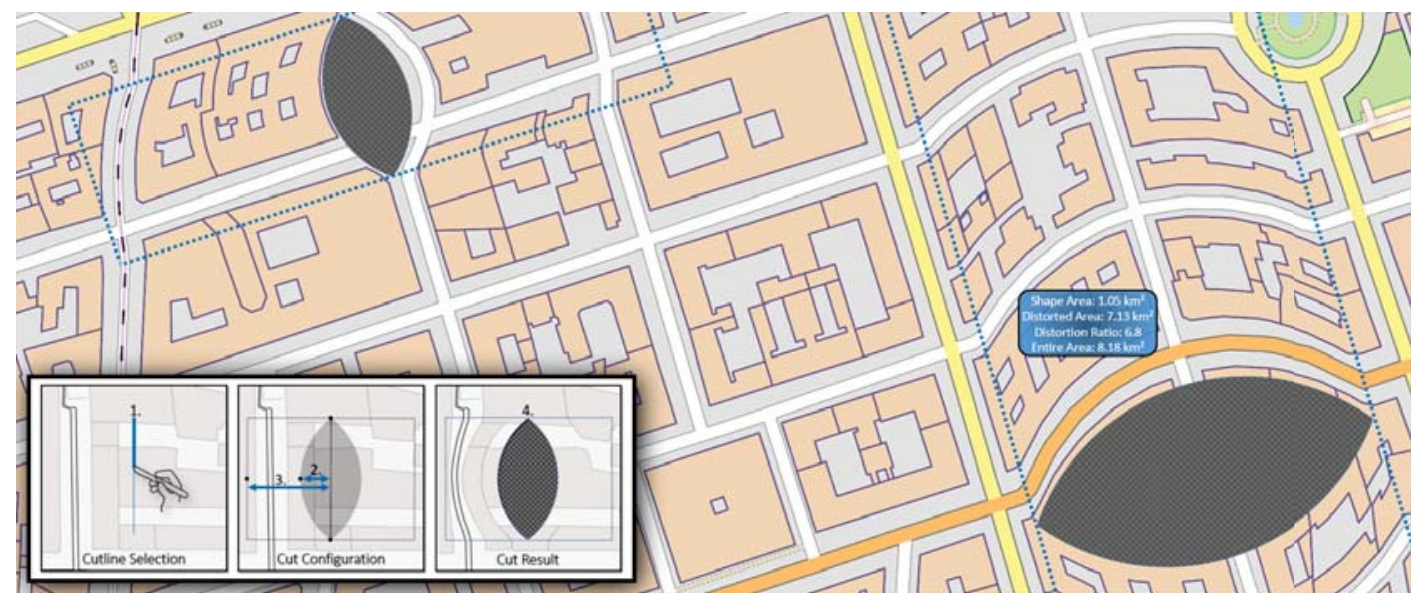

Figure 1: SurgeryCuts allows to create arbitrary cuts on maps for additional canvas area. This example shows two cuts in different size and orientation. The outlined area is the area affected by distortion. The inset explains the interactive configuration and creation of a cut.

\begin{abstract}
Visualizing contextual information to a map often comes at the expense of overplotting issues. Especially for use cases with relevant map features in the immediate vicinity of an information to add, occlusion of the relevant map context should be avoided. We present SurgeryCuts, a map manipulation technique for the creation of additional canvas area for contextual visualizations on maps. SurgeryCuts is occlusion-free and does not shift, zoom or alter the map viewport. Instead, relevant parts of the map can be cut apart. The affected area is controlledly distorted using a parameterizable warping function fading out the map distortion depending on the distance to the cut. We define extended metrics for our approach and compare to related approaches. As well, we demonstrate the applicability of our approach at the example of tangible use cases and a comparative user study.
\end{abstract}

\section{Scope of SurgeryCuts}

Geographic maps are representations of spatial contexts, as MacEachren explains in "How maps work" [Mac04]. Using maps, information can be tied and connected to single locations or extensive areas. Enriching geographical maps with additional information is both a common and well-researched task. Yet, this task can also prove to be challenging: Inherently, information that you want to add to a specific location has to be drawn on top of the map or taken out of the immediate spatial context by putting it next to the map. At the same time, anything you add on top of a map also occludes what lies beneath. As a result, map context and information can get lost. A variety of techniques has been developed to alleviate this problem. We have identified three families of approaches that can be applied to a map in order to add new or substantiate existing information which are relevant to our proposal. We compare them in Figure 2.

Occlusion-Based First, we regard occlusion-based solutions that solely rely on imposing information on the map without altering the map structure. Due to the concept's simplicity, the field 


\begin{tabular}{|c|c|c|c|c|c|c|c|}
\hline \multirow[b]{2}{*}{ 1. SurgeryCuts } & \multirow{2}{*}{$\begin{array}{l}\text { ACA } \\
\text { Yes }\end{array}$} & $\begin{array}{l}\text { Structure } \\
\text { Independence }\end{array}$ & Viewport Shift & \multirow{2}{*}{$\begin{array}{l}\text { Supported } \\
\text { Cardinality }\end{array}$} & \multicolumn{2}{|c|}{ Distortion (Intensity: I, Direction: D) } & \multirow{2}{*}{$\begin{array}{l}\text { References \& } \\
\text { Examples }\end{array}$} \\
\hline & & Yes & No & & Intensity: Controlled & Direction: Directional & \\
\hline 2. SpaceCuts & Yes & No & Yes & Low & \multicolumn{2}{|l|}{ None } & [BJSK16] \\
\hline 3. Volume Manipulation & Yes & Partially & does not apply & Medium & Intensity: Controlled & Direction: Non-Uniform & [Csc06] \\
\hline 4. Route-Zooming & Yes & No & Yes (Topology-Dependent) & Medium & Intensity: Topology-Based & Direction: Topology-Based & [SLQW17] \\
\hline 5. Bifocal Display & No & No & No & Low & Intensity: Topology-Based & Direction: Topology-Based & [SA82,MRC91] \\
\hline 6. Continuous Lens (Fisheye) & No & Yes & Depends - mostly no & Low & Intensity: Controlled & Direction: Uniform & [Fur86,SB94] \\
\hline $\begin{array}{l}\text { 7. Discrete Lens (Interactive) } \\
\text { \& Focus+Context }\end{array}$ & No & Partially & Depends - mostly no & Low & Intensity: Domain-Dependent & Direction: Non-Uniform & $\begin{array}{c}\text { [PPCP12, } \\
\text { TGK*17 YOT09] }\end{array}$ \\
\hline 8. Ray Deflectors & No & Yes & Depends on perspective & Low & Intensity: Controlled & Direction: Uniform & [KY95] \\
\hline$x$ & No & Yes & No & High & \multicolumn{2}{|l|}{ None } & $\begin{array}{l}\text { [AAB*13,DMK05, } \\
\text { Mac04] }\end{array}$ \\
\hline
\end{tabular}

Figure 2: A comparison of techniques related to SurgeryCuts and examples for each kind of approach.

of resulting approaches is vast and cannot be discussed in detail. MacEachren [Mac04] provides an introduction to the representation of information on maps, and an extensive display of data visualization techniques for maps can be taken from Dykes et al. [DMK05].

Distortion-Based Second, distortion-based approaches can alleviate the impact of occlusion by distorting a map, emphasizing areas and presenting more or different information than compared to the undistorted original scale of the map. A recent survey by Tominski et al. [TGK ${ }^{*} 17$ ] provides an extensive overview on the current approaches. Classical distortion-based techniques such as the graphical Fisheye Views introduced by Sarkar and Brown [SB94] mostly employ a uniform distortion, such as a circle fading out the distortion radially around the center of focus. On the other hand, discrete lenses can be arbitrarily shaped and do not necessarily follow uniform distortion patterns. For discrete lenses, it can even happen that a non-regular distortion shape causes overplotting again, as examples of the survey of Neumann and Carpendale [NC03] show. A specific technique to be mentioned are Bifocal Displays, as introduced by Spence and Apperley [SA82] in 1982. Here, a portion of the data space is presented in more detail than another. While retaining continuity across boundaries, the area not in focus is distorted to adapt to the additional space needs of the detailed view. The focus+glue+context approach by Takahashi [Tak08] can be considered as a combination of Fisheye Views and Bifocal Displays. Other techniques exist in the field of volume distortion, such as the Ray Deflector approach by Kurzion and Yagel [KY95], which share aspects with SurgeryCuts concerning selectivity and locality of introduced deformations. While we focus on 2D map manipulation only, ray deflectors also do not regard the creation of additional space on the map or implications for the surrounding elements.

Structural The same metaphore of surgical cuts, although in a much more literal way, is employed by Correa et al. [CSC06], who describe a set of methods for the interactive manipulation of volume data to create illustrative visualizations. Extending a cut to enable observation of underlying tissue in a human body rendering is one of the prominent examples. Conceptually related, their work is oriented towards interaction with volumes, whereas we focus on the creation of the additional canvas area and the implications of the distortion towards the spatial map topology. Finally, a set of rather recent approaches including the SpaceCuts approach by Buchmüller et al. [BJSK16] and Route-Zooming as presented by Sun et al. [SLQW17] aims to make use of map structures such as roads or railroad tracks. By expanding these structures in a controlled manner, additional canvas areas can be generated without occluding other map features. Haunert et al. [HS11] combine structural dependency and distortion techniques to highlight certain areas of road networks but do not create additional space for visualization. Necklace Maps by Speckmann and Verbeek [SV10] arrange additional information around geospatial areas, which requires having enough empty or unimportant space around the features to be visualized.

Further techniques exist to contextualize information with spatial locations outside of a given map. For example, off-screen visualizations such as EdgeRadar [GI07] place additional information around a map location. Since the focus of SurgeryCuts is to create additional visualization space directly on a map, we intentionally do not compare to techniques that pull the visualization context out of the map. For each of the techniques we take into account, we have identified a set of five relevant features that can be employed to characterize its implications for the physical map space and the potential and consequences of adding information to the map. We compare the following aspects:

ACA is an Additional Canvas Area. ACAs indicate whether a technique creates additional room on a map that does not interfere with map features and that can be used for applying visualizations.

STRUCTURE INDEPENDENCE denotes whether a technique can be applied to arbitrary parts of a map or it is tied to fixed map structures such as roads or rivers.

VIEWPORT SHIFT occurs if a technique alters the map in a way that not all parts of the map included in the original viewport will be displayed after application of the technique.

CARDINALITY indicates the amount of information a technique potentially allows to additionally put on a map.

DISTORTION denotes whether the scale of the map is changed 
by an approach. If so, the intensity denotes how the amount of distortion introduced is determined. Also, direction explains how the distortion is distributed on the map.

While each of the presented techniques has individual strengths and suitable use cases, comparing the properties of each approach reveals a gap that remains to be filled: Occlusion-based solutions and lenses do not provide extra space for visualizations on the data space. While occlusion-based approaches just plot over existing map features, lenses and bifocal displays also add distortion to a map. Depending on the distortion technique (e.g., linear distortion vs. distortion based on predefined shapes or map features), a human's perception and cognition of the map can evidently suffer [Gut02], while still, map areas have to be occluded to add information. These issues are discussed extensively by Carpendale et al. [CCF97].

In contrast, some structural approaches aim to make use of map features to create an ACA that allow to put more information on a map without occluding surrounding map features. The SpaceCuts approach by Buchmüller et al. [BJSK16] proposes to cut a map along continuous map features such as a rivers in two or more pieces, then pull the map apart along the created cut axes to create an ACA. Yet, the amount of created space per distance unit depends on the angle towards the axis between the endpoints of the cut - in other words, depending on the cut shape, sometimes no additional area is created at all and sometimes even occlusion can happen when the map is pulled apart. Further, the cut has to stretch through the whole viewport in order to be able to be pulled apart. Inherently, this technique causes map content to be shifted outside of the viewport when a cut is pulled apart. Sun et al. [SLQW17] present another technique capable of creating an ACA. The Route-Zooming approach is based on the application of seam-carving to a map. Using complex shifting and distortion operations, a map feature such as a street can be expanded to create an ACA. The resulting distortion and displacement is non-linear and aims to retain the topology of the street networks used by the authors to demonstrate their approach.

The principle of SurgeryCuts is the introduction of cuts in a map at a position where additional information is to be visualized. Figure 1 shows an illustration of the process. Much like a surgeon would do, the cut area is extended by pulling the sides of the cut outwards orthogonally to the cutline. Finally, the distortion introduced to the map by this operation will be gradually faded out until the original map state is re-established. Consequently, a SurgeryCut only introduces local, controlled changes to the map that can be parameterized (e.g., by changing the area influenced by the cut).

SurgeryCuts Contribution SurgeryCuts introduces the following key novelties with respect to the discussed available proposals.

- It has the unique feature of visualizing additional information on any map area without a) occluding map features, b) changing or shifting the viewport of the map, or c) relying on map structures like roads or rivers. Moreover it allows for explicitly fine tuning the distorted area size and orientation.

- Its intended usage differs from techniques that inspired authors, like Focus plus Context $(\mathrm{F}+\mathrm{C})$ or illustrative visualization. The purpose of SurgeryCut is to add additional information on the map, information that refers to elements close to the cut point, making each cut specific to its location, static, and tailored to the surrounding by setting distortion orientation and extent. Conversely, $\mathrm{F}+\mathrm{C}$ techniques are inherently dynamic, do not create new space, and allow for exploring the visualization, e.g., moving a magnifying lens on the map and zooming to provide more details. Illustrative information techniques manipulate volume models and deal with layered planes of information with the main goal of making evident relative positions among elements of different layers, e.g., human body parts below a cut of the skin.

- To the best of the authors' knowledge, no lens proposal introduces new space on the map and, as a consequence, using a lens formalism requires to reformulate it in a possibly not straightforward way to take into account the new space that require additional parameters and maths. SurgeryCuts inherently features a clear boundary between added area and map, whereas with purely distortion-based approaches, this distinction is not so sharp. Consequently, with conventional approaches, an additional consideration step concerning overplotted areas is required.

Moreover, SurgeryCuts comes with several quality metrics that allow for steering the technique and comparing it with existing solutions; tangible use cases and a controlled user study demonstrate the applicability and the advantages of the technique.

Paper structure The details and implications of the construction of a SurgeryCut is explained in Section 2. Section 3 lists a set of metrics derived from the cuts and explains their calculation. These metrics will later be used for comparison to other techniques in Section 4. Section 5 discusses cases that benefit from the usage of SurgeryCuts and illustrates the applicability thorough two practical use cases from the domains of network security and subway exit scenario. Section 6 describe a controlled experiment challenging our solution against an occlusion based technique and, finally, Section 8 provides a discussion of SurgeryCuts with regard to the scope of the technique and advantages and shortcomings.

\section{Technique}

In principle, the construction of a SurgeryCut can be summarized in three basic steps shown in Figure 1. First, identify a suitable cutline position (1), according to the requirements of a particular use case. Second, the extent of the cut shape (2) and the area of the map influenced by the cut (3) should be set. Lastly, the SurgeryCut is applied to the original map, while introducing distortion only to the influence area (4), and not to the surrounding context. The overall map scope remains the same, while distortion can be controlled.

In the following, we want to give an in-depth explanation of the theoretical and practical aspects of this process. First, in Section 2.1 we discuss the implications of the choice of geometric shape applied to distort the cut. As well, the distribution of the distortion is important to consider. Afterwards, we delimit necessary preconditions and introduce our strategy of vector displacement in Section 2.2.

\subsection{Shape Choice and Implications}

Before cutting a map area, it is necessary to make a decision on the shape to be created by extending the cut. A SurgeryCut follows three principles: First, the creation of a map should not alter the viewport of the unchanged map. Consequently, the map area influenced by a cut must be contained within the viewport. Second, the distorted area of the map should be generally minimized so that as much area as possible remains in the original representation. Third, the distortion result should retain map feature continuity as much as possible to 


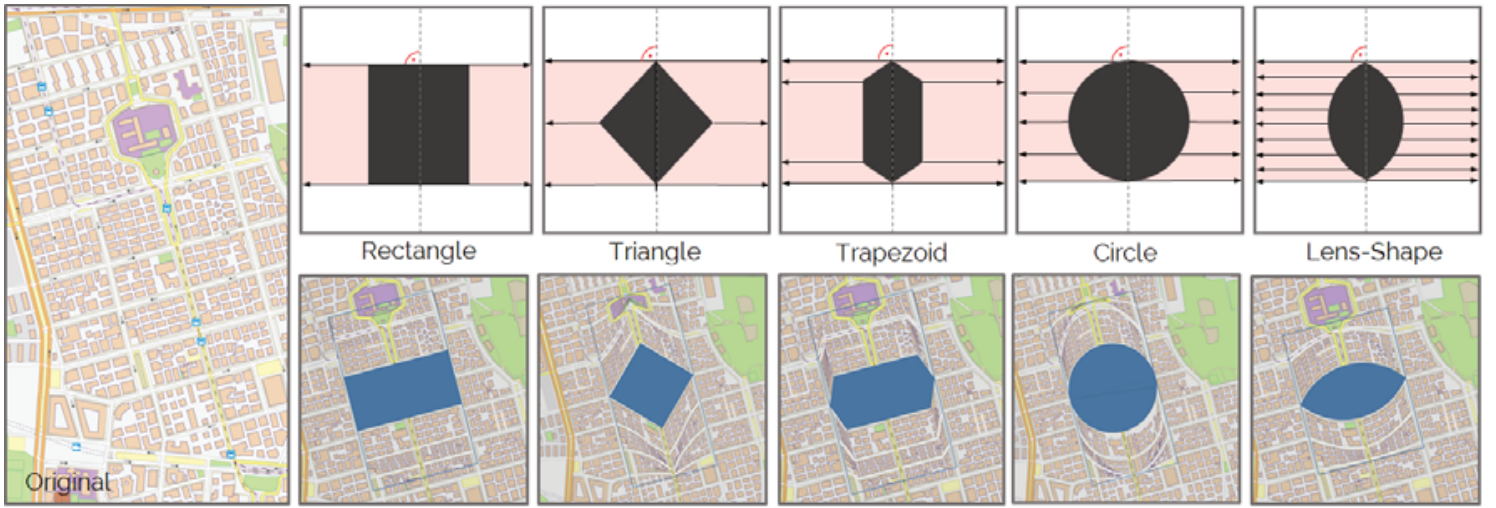

Figure 3: Five different shapes for the cut expansion. The distortion is orthogonal to the cutline, the length of the arrows indicates the amount of space available to distribute the amount of distortion. The arrangement of the arrows indicates breaking points in the amount of distortion introduced. Longer arrows indicate more space to distribute the distortion, thus introducing less intense changes on the map.

avoid losing spatial context. To exemplify different shapes and their implications, we compare five basic, regular cut shapes in Figure 3.

While theoretically, arbitrary, asymmetric cut shapes could be implemented, the resulting complex distortion would be very difficult to control and interpret, and relations and distances could be lost. Thus, we focus on symmetric shapes with the cut as the mirror axis. We exemplify five shapes in Figure 3: a rectangular shape, a triangle, a trapezoid, a circle and a lenticular shape for the ACA. We identify the number of turning points in the distortion gradient for each shape: While there are two such points in the rectangular shape, the triangle has three and the trapezoid four for each side of the cut. The circle and the lenticular shape feature have an infinite number of points where the distortion changes due to the linearity of their curvature. Naturally, we could distribute the introduced distortion along the vectors of the turning points. For example, in case of the circle, we could distribute the distortion all around. Yet, it is our goal to reduce the introduced distortion to a minimum. Thus, we choose to only distribute the distortion orthogonally to the cutline as indicated by the red area that denotes the distortion area when distortion is carried out orthogonal to the cutline. By choosing the cutline-orthogonal approach over the shape-edge distribution, the amount of distortion axes is limited to one affected axis of the map.

The application of each shape as cut form has unique implications to shape, size and configuration of the resulting distorted area. Under these prerequisites, we discuss three decision factors for the choice of suitable cut shapes in the following paragraphs. First, we consider the amount of distortion as well as the distortion progression along the cut as the distortion regularity. Second, we compare different configurations of the map topology around a cut to identify suitable cut shapes. Lastly, also the content to be placed in a cut is a decision factor for a shape to choose.

Cut Shape and Distortion Regularity First, we observe the nature of the space that has to be distorted towards the boundary and the transitions between distortion axes. The distance of each point along the shape to the boundary determines the amount of distortion necessary to compress the space taken by the cut. In Figure 3, this amount is represented by the length of the exemplary arrows placed at pivotal turning points on the shapes in the upper half of the image. For determining this amount, see also $\left|\overrightarrow{O O^{\prime}}\right|$ in Figure 4 and the supporting function 3 . While a rectangular cut shape is possibly best suitable as canvas for many different visualizations, the introduced distortion creates a very abrupt change in the visual map features at the borders of the shape, where everything is shifted left or right for the width of the rectangle. Perceptually, this would cause major disturbances in the map. As a result, in the worst case, an observer could not tell any more whether a map feature next to a cut is adversely distorted due to a sudden change of the cut shape or whether it is just the real shape of the map feature. The triangle and trapezoid shapes share this effect, since the change in distortion is quite distinct at the turning points. In comparison, the circular shape distributes the degree of distortion linearly along its outline, thus alleviating the effects of sudden changes. While this leads to a more homogeneous transition between distorted and undistorted map parts and within the distortion area, at the bottom and the top of the circle, it can happen that map features parallel to the gradient are absorbed. The fifth shape to compare is of lenticular nature, which avoids the corner cases of the circular shape, creating no abrupt directional distortions to map features along its outline and less structural issues at the top or bottom areas. A comparison of the five shapes applied as cuts to the same map excerpt can be seen in Figure 3 in the lower half. More details on the construction of a lens shape can be found below in Section 2.2.

Surrounding Area Topology The second factor to consider when choosing an appropriate shape is the topology of the area surrounding a cut. While conceptually applicably to any kind of map, especially dense urban surroundings pose challenges when a cut is introduced: The map displacement corresponds with the shape of the cut. In other words, while the topology, e.g., the street networks, is not destroyed, it still gets distorted, coming at the cost of interfering with an observer's expectations of a certain area. The choice of shape determines the perception of the resulting map. For example, a round cut shape such as the circle or the lenticular cut creates a more prominent affected area in a regular street grid, e.g. like in many US cities, than in less uniform street grids of European cities, since the cut disturbs the parallel order of the street network.

Coming to the question which cut shape is suitable best for which kind of surrounding area topology, the answer is use-case dependent. If the goal is to minimize map disturbance, it is prudent to chose 
a shape which corresponds with the alignments of the main map features in the surrounding area. For example, if the surrounding features such as streets are mostly aligned orthogonally, choosing a rectangular cut would fit to the street layout, but only if parallel to the other features. On the other hand, the cut would then create a transition-free distortion, potentially shifting whole blocks away from their original context. Consequently, the ability of users to navigate the resulting map would suffer if map features are shifted without clearly seeing how and by how much. Alternatively, one can argue that deliberately choosing a rounded cut shape for an orthogonally built street network works better since a user is then able to attribute the rounding of the distorted features to the distortion and not the original map situation, thus being able to retain the right notion of the real map topology when looking at the distorted one. We alleviate such situations by providing visual cues marking the outlines of the created cut and its area, and by fading out the distortion outwardly towards the boundaries of the affected area.

Cut Content The third decision factor for the cut shape is the intended content to be placed in it. Thinking in terms of Data-Ink Ratio, a cut should be as small as possible to convey the information needed in order to minimize the map distortion. Thus, the cut shape ideally matches the shape of the content placed within the cut. Yet, not all types of content can be aligned exactly with a cut shape. Icons, glyphs, labels and visualizations often are not of regular nature.

Further, if a related visualization element is to be placed outside of the cut, shape and relation of the outer element could be determined by the cut. Especially, if any outside visualization with spatial relation would fall within the distortion area of the cut, it would be subject to the same distortions applied to the map beneath.

Shape Choice The cut shape choice depends on several variables and requirements, like the placement of the surrounding map features such as streets and their alignment and parallelism, the size of the cut, its orientation, and the desired content. Concerning the latter, the shape optimal for the desired cut content can potentially be a different one than what would be appropriate concerning the surrounding area. Consequently, the cut shape choice can only follow a thorough assessment of requirements and map surroundings. We aid a user in doing so by providing contextual information about a cut during the creation procedure, such as the area of the generated ACA against the distorted area created as shown in Figure 1. In summary, considering the three factors, we come to the conclusion that the shape choice is very much use-case dependent and must always be the result of careful consideration. In some use cases, it is more important to distribute the distortion more evenly, while in others, retaining the map appearance is preferable.

While we do not recommend any shape over another, the lenticular shape combines most aspects of other regular shapes in terms of constructibility and distortion distribution. Thus, the lens shape is suitable as a representative for the many other possibilities. As well, using a lenticular shape, we fulfill the recommendations defined by Carpendale et al. [CCF97] when designing distorted views:

1. We avoid occlusion completely.

2. By providing control over the affected distortion area, we keep focal points close to their original location.

3. The continuous distortion function of the lens shape creates a smooth integration of the ACA into the map.
4. The lens shape is a familiar distortion curve.

5. By restricting the distortion to a single axis orthogonal to a cutline, we are maintaining orthogonality and the mental map as far as possible, helping users to retain a clear idea of the affected area.

6. While the cut is not yet animated, we intend to explore this possibility in future work.

Consequently we will explain the construction and application of SurgeryCuts using the lenticular shape in the remainder of this publication, bearing in mind that the construction of other regular shapes follows the same principle.

\subsection{Lenticular SurgeryCuts: Theoretical Specification}

In this subsection we provide a theoretical frame for the geometry definition of lenticular SurgeryCuts, i.e., a precise and systemindependent basis for the description of our vector displacement (in Subsection 2.2.2) and distortion metrics (in Section 3). We initially considered to rely on the general magnifying approach proposed by Carpendale and Montagnese in [CM01] that uses a reference view plane to displace elements in the representation plane to magnify the image in the focus and to accommodate distortion; however, we have decided to not use that proposal because it always implies the presence of a magnification that does not exist in our approach. Standard radial distortion techniques having an empty center can deal in a simpler way with our cut formalization but are not able to cope with all the details we need to fine tune our technique and that solution can be considered as a means for a faster implementation.

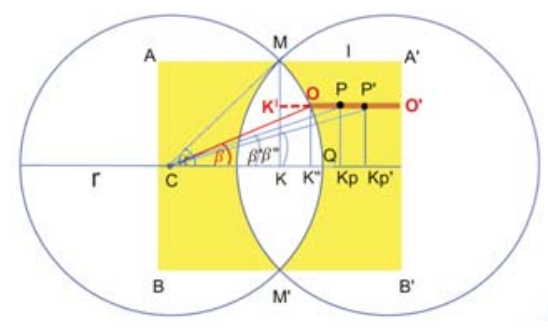

Figure 4: A lenticular SurgeryCut. The yellow area represents the distorted space, the inner white area represents the additional canvas area, ACA, produced by the cut, while the rest of the space is unchanged. Distortion is not constant: for $\beta=\alpha$ is 0 and for $\beta=0$ it exhibits the maximum value.

\subsubsection{Geometry Definition}

The process of generating a SurgeryCut encompasses calculations mainly within one vector space $\mathbf{P}$. Thus, this space yields coordinates for both cut shapes and map geometries. We define $\mathbf{P}:=\left[x_{1}, x_{2}\right] \times\left[y_{1}, y_{2}\right]$ with $\mathbf{P} \subset \mathbb{R}^{2}$ as a Cartesian space, illustrating a two-dimensional surface. Additionally, we define that a position vector $P \in \mathbf{P}$ lies within a canvas' field of view and $P$ is given in projected (cartographic) coordinates. For instance at our developed demonstrator, these coordinates are determined by an UTM projection and stem originally from (latitude, longitude) tuples. For more details on our demonstrator, see the introduction of Section 5.

The potentially interactive construction of a SurgeryCut starts by defining two distinct position vectors $M, M^{\prime} \in \mathbf{P}$, which describe the end points of the basic cutline, see Figure 4. Since our approach allows shapes of arbitrary rotation, the following formalization enables a rotation-invariant and unambiguous geometry definition. We 
assume $M$ to be positioned more on the right. If $M$ and $M^{\prime}$ share the same $x$-coordinates, we focus the vectors' $y$-coordinates: So, it holds either $M_{x}>M_{x}^{\prime}$ or $M_{y}>M_{y}^{\prime}$, the latter namely in case of $M_{x}=M_{x}^{\prime}$.

Subsequently, a rectangular area is centered around the cutline which will absorb the distortion, implied by the SurgeryCut. We focus the cutline's normal to calculate this area's boundary vectors $A, A^{\prime}, B$ and $B^{\prime}$ in Figure 4. With $X \in\left\{M, M^{\prime}\right\}$ and a fixed $l \in \mathbb{R}_{>0}$, we can define these position vectors by

$$
X \pm l \cdot\|\vec{n}\| \quad \text { with } \quad \vec{n}=\left(\begin{array}{c}
-\left(\overrightarrow{M M^{\prime}}\right)_{y} \\
\left(\overrightarrow{M M^{\prime}}\right)_{x}
\end{array}\right)
$$

Finally, for the ACA construction we can think of two $r$-sized circles centered at $C, C^{\prime}$ with $C \neq C^{\prime}$. Now let the circle boundaries touch the position vectors $M$ and $M^{\prime}$ : then, the actual ACA is given by the circles' intersection area, implying a symmetry around the cutline. At ACA construction we require particular properties: with $K$ being the cutline center $\frac{M+M^{\prime}}{2}$, it needs to hold

1. $|\overrightarrow{K Q}| \in(0, r]$, where $Q$ is one of the two position vectors on the ACA outline which maximize the cutline distance. That is, the ACA has non-zero extent and could theoretically represent at most a full circle. Note that this first property can be seen as an implication of mentioned cutline and circle geometry settings.

2. $2|\overrightarrow{K Q}| \leq l$. That is, the ACA does not exceed the rectangle $\left(A, A^{\prime}, B^{\prime}, B\right)$ and there is enough space to absorb distortion. The factor 2 is essential for position vectors which are originally located in the ACA.

\subsubsection{Vector Displacement}

Like in Figure 4, we assume a $P \in \mathbf{P}$ in the unchanged space, and we want to determine the position vector $P^{\prime}$ in changed space. For this we apply a scaled version of the cutline's normal to $P$. In the following we contour the three relevant scaling functions below, while using notations from Figure 4.

$$
P^{\prime}=P+t(P) \cdot w(P) \cdot s(P) \cdot\|\vec{n}\|
$$

First, we define $t: \mathbf{P} \rightarrow[0,|\overrightarrow{K Q}|]$ for initial translation scalars:

$$
t(P):= \begin{cases}0 & \text { if } P \text { is outside }\left(A, A^{\prime}, B^{\prime}, B\right) \\ \left|\overrightarrow{K^{\prime} O}\right|=r \cdot[\cos (\beta)-\cos (\alpha)] & \text { else }\end{cases}
$$

Obviously, each position vector outside $\left(A, A^{\prime}, B^{\prime}, B\right)$ remains entirely untouched. Note that we can derive case 2 of function $t$ via

$$
|\overrightarrow{K Q}|-\left|\overrightarrow{K^{\prime \prime} Q}\right|=[r-r \cdot \cos (\alpha)]-[r-r \cdot \cos (\beta)]
$$

Next, we linearly weight the obtained translation scalars by $w: \mathbf{P} \rightarrow$ $[0,1]$. This function is responsible for a decreased scalar, as a position vector is more distant from the cutline.

Thus, a smooth integration of the changed space into its surrounding context gets ensured. In addition, $w$ avoids that a $P$ inside $\left(A, A^{\prime}, B^{\prime}, B\right)$ gets shifted outside this region, which could lead to occlusion or other side effects in the surrounding space. So, by this property and the first case of function $t$, we ensure the basis for one contribution of our paper: original viewport preservation.

$$
w(P):= \begin{cases}1 & \text { if } P \text { is inside or touches the ACA } \\ \frac{l-\left|\overrightarrow{K^{\prime} P}\right|}{l-\left|\overrightarrow{K^{\prime} O}\right|} & \text { else }\end{cases}
$$

Finally, the integer-valued function $s: \mathbf{P} \rightarrow[-1,1]$ determines a translation scalar's sign. The sign depends on which side of the cutline a $P$ is located in unchanged space. This function is definable through multiple strategies: for instance, one could (a) consider the sign of the cross product $\overrightarrow{M M^{\prime}} \times \overrightarrow{M P}$ or (b) split $\left(A, A^{\prime}, B^{\prime}, B\right)$ and check for point containment. Clearly, our rotation-invariant formalization in Subsection 2.2.1 can support any strategies. Note that a special case needs to be handled: point vectors directly located on the cutline. To avoid unclear translation directions, we recommend tiny translations by $\varepsilon \cdot\|\vec{n}\|$ as part of map feature preprocessing. The visual impact is minimal, but in particular the mentioned case is very unlikely to even occur at real-valued spaces, common geographic data models (e.g. DIME,TIGER) and (interactive) cut construction.

\section{Quality Metrics}

In line with the general idea of Visual Quality Metrics (see, e.g., Bertini et al. [BS06]) we provide a set of metrics for evaluating or steering our proposal. This section introduces the metrics referring to the lenticular cut shape (see, Figure 3) but it is straightforward to extend them to other shapes. To simplify the comparison with other techniques, metrics are formulated using points (instead of vectors), length of lines, and angles between them. Essential cut parameters are the circles radius $r=C Q$, the compressed area length $M A^{\prime}$ (i.e., $l$ ), and the angle $\alpha$ (see Figure 4). The introduced metrics have the main goal of supporting three main activities:

- Characterize the image quality after the cut;

- Compare SurgeryCuts with other techniques;

- Steer optimization strategies.

According to these objectives we designed the following metrics: ACA. Additional Canvas Area made available on the image. This metric calculates how much room a cut makes. Referring to Figure 4 , the additional canvas area is:

$$
A C A(r, \alpha)=2 \cdot\left[r^{2} \alpha-h \cdot C K\right]=2 r^{2} \cdot[\alpha-\sin (\alpha) \cdot \cos (\alpha)]
$$

where $h=r \cdot \sin (\alpha)$ and $C K=r \cdot \cos (\alpha)$.

Area affected by distortion. This metric computes the area that is bearing distortion to accommodate the ACA.

$$
\text { DistortedArea }\left(r, \alpha, M A^{\prime}\right)=2 \cdot M A^{\prime} \cdot 2 h-A C A(r, \alpha)
$$

Ratio between distorted area and ACA (the lower the better). This metric calculates the penalty we have to pay for the ACA:

$$
\text { DistortedAreaACARatio }\left(r, \alpha, M A^{\prime}\right)=\frac{\text { DistortedArea }\left(r, \alpha, M A^{\prime}\right)}{A C A(r, \alpha)}
$$

The above metrics provide for an overall judgment of the performance of SurgeryCuts, the introduced distortion, and trade-off between ACA and distorted area. In the following we provide means for measuring the point-wise impact of a cut, measuring how a specific point or a specific area is affected by the technique.

Linear distortion, LD. We characterize the intensity of the linear distortion that varies according to $\beta$ (no distortion for $\beta=\alpha$, maximum distortion for $\beta=0$ ) as:

$$
L D\left(r, \alpha, \beta, M A^{\prime}\right)=\frac{K^{\prime} O}{M A^{\prime}}=\frac{r \cdot[\cos (\beta)-\cos (\alpha)]}{M A^{\prime}}
$$


The formula provides the distortion for a single point $P$; if we want to compute the overall distortion along an arbitrary interval $\left(\beta_{1}, \beta_{2}\right)$ of the distorted area we use the following formula:

$$
\operatorname{Distortion}\left(r, \alpha, M A^{\prime}, \beta_{1}, \beta_{2}\right)=\int_{\beta_{1}}^{\beta_{2}} \frac{r \cdot[\cos (\beta)-\cos (\alpha)]}{M A^{\prime}} \delta \beta
$$

Displacement, DSP. When considering a single point $P$ in the distorted area (outside the distorted area the displacement is zero, see Function 3) we characterize its displacement $\left(P P^{\prime}\right)$ as follows:

$$
D S P\left(r, \alpha, \beta, M A^{\prime}, K^{\prime} P\right)=r \cdot[\cos (\beta)-\cos (\alpha)]\left[1-\frac{K^{\prime} P}{M A^{\prime}}\right]
$$

Bearing Deviation, BD. This metric calculates the bearing deviation, for a single point $P,\left(\beta^{\prime}-\beta^{\prime \prime}\right)$ with respect to the center of the cut, as follows :

$$
\begin{aligned}
B D\left(r, \alpha, \beta, K^{\prime} P\right)= & \\
& \arcsin \left(\frac{r \cdot \sin (\beta)}{C K_{p}}\right)-\arcsin \left(\frac{r \cdot \sin (\beta)}{C K_{p^{\prime}}}\right)
\end{aligned}
$$

where $P K_{p}=P^{\prime} K_{p^{\prime}}=O K^{\prime \prime}=r \cdot \sin (\beta)$.

SurgeryCuts can be used in two different modalities (see Section 5): automatic or manual. The automatic mode is intended for users or applications that want to add some information on a map and leaves to the system the burden of opening the cut able to accommodate the new information; manual mode leaves to the user the full control of the cut parameters. One straightforward application of the above metrics in such context is to steer/automate the cut process: in manual mode the user can design the cut observing the actual values of the metrics, thus supporting his or her decisions on cut parameters (e.g. the compressed area length $M A^{\prime}$ ); in automatic mode, the user can set some quality thresholds (e.g., maximumDisplacement, maximumLinearDistortion, etc.), having the system automatically computing the cut parameters that accommodate the object to be placed in the map respecting the thresholds. It is worth noting that this complements the general choice of the cut orientation: having numerical measures on the distortion and bearing allows for designing automated techniques able to select the cut orientation that, using the same quality thresholds, minimize the distortion of relevant areas (e.g., buildings, streets, etc.), distributing the distortion on less relevant areas (e.g., lakes, forests, etc.)

Another application is using the metrics to maintain spatial relationships between the object inserted in the cut and the surrounding context. For example, assume we put tabular information on a metro station and want to show the 5, 10 or 15 minutes walking distance to places around that station, distances that are represented by a polyline centered on the station. After the cut, the polyline requires to be adapted to point displacement to preserve its original meaning.

\section{Comparison}

This section presents evaluation activities that have been carried out on SurgeryCuts. In particular, SurgeryCuts we compared to related techniques, benchmarking their performance using the metrics introduced in Section 3. Techniques closest to SurgeryCuts are SpaceCuts [BJSK16] and Route-Zooming [SLQW17]: both
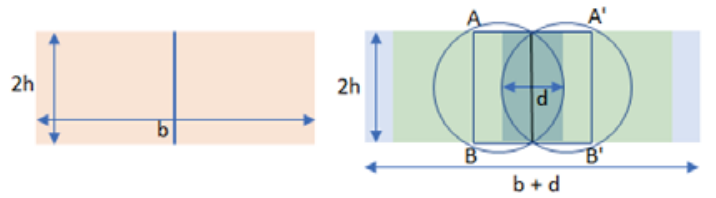

Figure 5: Comparing SurgeryCuts with SpaceCuts.

share the distinctive feature of creating an ACA that does not interfere with map features; however, Route-Zooming only deals with routes, disregarding any other map feature and both SpaceCuts and Route-Zooming rely on the presence of a feature that, in the case of SpaceCuts, must traverse the whole map.

SurgeryCuts - SpaceCuts In Figure 5 (left), we abstract the behavior of SpaceCuts using as feature a vertical line (that represents the optimal case for SpaceCuts, maximizing the ACA) that is enlarged of $d$, creating an ACA of size $2 h \cdot d$. That requires to displace the whole areas on the left and right of the feature and extending the viewport of $h \cdot d$ areas on the left and on the right.

In the right image of Figure 5 the result of this activity is displayed: the dark area in the middle is the ACA produced by SpaceCuts, the green areas represent the displacement of $d / 2$ and the light blue area represents the part of the image that is outside the original canvas. To compare such results with SurgeryCuts, we have used a cut of height $2 h$ centered in the middle of the SpaceCut with a maximum displacement of $d / 2$, see Figure 5 , right image. It opens an ACA that is comparable to SpaceCuts $(\approx 20 \%$ less $)$ and produces a distorted area of $2 h \cdot A A^{\prime}-A C A(r, \alpha)$, contained in the rectangle $\left(A, A^{\prime}, B^{\prime}, B\right)$ with a maximal point displacement of $d / 2$. The DistortedAreaACARatio (the lower the better) for SurgeryCuts is about 1.6, i.e. 15 times less than SpaceCuts, and 22 times less if we consider the extended canvas.

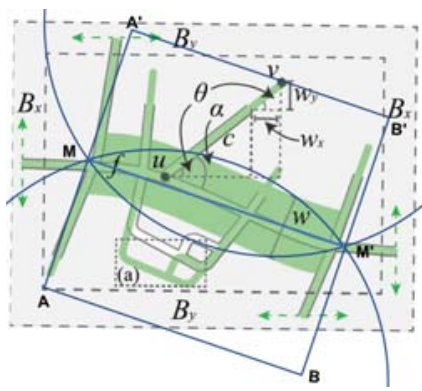

Figure 6: Comparing SurgeryCuts with Route-Zooming. The background image has been taken from [SLQW17].

SurgeryCuts - Route-Zooming Figure 6 shows a comparison between SurgeryCuts and Route-Zooming. Route-Zooming creates an ACA of size $M M^{\prime} \cdot w$ on the context road $f$, while displacing surrounding roads within the bounding box $B_{x}, B_{y}$. We assume the optimal case for Route-Zooming in which the actual context road does not intersect other context roads, and that all displacements happen within the bounding box. In this case we have selected a cut with direction and length equal to those of the contextual road and, because Route-Zooming does not propagate $w$ displacement across the whole image, we have set the cut maximumDisplacement greater than $w$ to produce an ACA with the same size of Route-Zooming 
one. As well, we set $M A$ to about $2 w$ : this smoothly accommodates all road displacements (until the furthest displaced point $V$ ), still presenting a DistortedAreaACARatio 7\% lower than Route-Zooming. Moreover, SurgeryCuts provide means for preserving also features close to the roads while Route-Zooming completely neglects them.

As a conclusion, we can say that SurgeryCuts is able to provide comparable ACA with respect to SpaceCuts and Route-Zooming, without shifting the image viewport and with a better or comparable trade-off DistortedAreaACARatio. Moreover SurgeryCuts is totally independent from map features (with respect to SpaceCuts) or roads (with respect to Route-Zooming) and deals with the whole map.

\section{Application Scenarios}

In the following, we describe the two main classes of use cases that benefit most from the application of SurgeyCuts.

1. The first class addresses the goal of adding details to an element of the map, details that are somehow connected to other map locations: in this case the cut contains an enriched representation of the original object and optional links allow for connecting the enriched element to other map locations. Examples are expanding a $3 \mathrm{G} / 4 \mathrm{G}$ telecommunication antenna making evident its internal characteristics and showing the orientation of its radio signal, or expanding an element of a broadband fiber optic network, showing its communication capability and linking it to its terminal points and to the backbone.

2. The second class of applications is the situation in which the cut designer wants to add some additional information that refers to a group of elements placed in a map area. Instead of labeling each element with additional text, a central cut in the area of interest allows to collect the needed information without occluding the map. Additional links can be added on the map, connecting the new information to the elements it refers to. Typical tasks include searching and comparing elements based on the added information like, e.g., adding temporal information about the red/green state of traffic lights in a high traffic area, or collecting aggregated information about the characteristics of the restaurants in a specific neighborhood to support a user's dining decision.

The next two subsections exemplify the aforementioned classes with two applications of SurgeryCuts: one belonging to the first class, detailing sub-networks nodes with internal connectivity (see Subsection 5.1), and another one adding contextual information to a set of subway exits (see Subsection 5.2). Both scenarios are built using our SurgeryCuts demonstrator program: after a user-steered import of arbitrary, XML-based OpenStreetMap vector data, the demonstrator enables interactive cut sketching and the map transformation relies on the identification of intersecting features and internal map modifications (e.g., line segmentation for smooth transitions, feature displacement as in Subsection 2.2.2). We can render and export transformed maps along with annotated cut coordinates, and subsequently handle SurgeryCuts in arbitrary environments.

\subsection{Critical Infrastructure Cyber-Protection}

The first application scenario regards the monitoring, analysis and cyber-defense of a critical infrastructure SCADA network for power distribution from cyber threats, based on what was developed in the European project "Panoptesec" [Pan16]. One of the main tasks to support is the representation of the network topology and components, for most of them requiring to elicit their relation with respect to their geographical position and controlled SCADA devices. The rationale behind it is that most of these components remotely govern a power distribution cabin present in a particular area (e.g., a power generator) and the eventual impairment of one of them has consequences on the related geographical area. It is common to prioritize the monitor and intervention activities depending on the particular served area, and to analyze the network status from both geographical and logical perspectives. Problems arise when a computer sub-network must be geo-localized (many computers share the same geographical position, e.g., a building containing a data-center composed of many appliances) and network connections could be lost due to use of visual abstraction for representing the network or contextual information could get lost due to superimposition of this information on the map. At the same time is possible to represent the network topology using common node-link diagram representation, losing at that point lots of information about geographical positions. Existing solutions, see, e.g. [AS17], rely on multi-coordinated visualizations, one representing the network topology and another its geographical spread, forcing a security operator to reason on one perspective at a time. Additionally, even considering coordination between views, the difference in layout between the geographical and topological obstacles the analysis, asking for frequent disruption in the analysis process due to switching between the different layouts. To overcome these drawbacks we applied SurgeryCuts, see

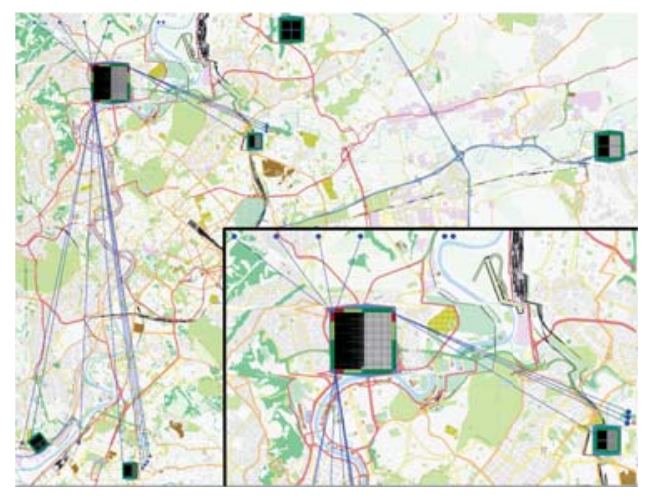

Figure 7: Applying SurgeryCuts to a power distribution network: all the multi-node glyphs of sub-networks are accommodated in specific cuts, keeping both the whole network and contextual information visible. A detail of one of the glyph is magnified in the bottom-right corner. Being the inserted information a square matrix we used a trapezoid-shape cut that better fits w.r.t. the lenticular-shape

Figure 7, creating different cuts on the map, each of them accommodating a matrix-like glyph representing the sub-network nodes connectivity. It allows the security operator to get the complete overview of the network topology and reachability, and the relations between logical nodes and connected physical SCADA appliances in the same view, without relying on any additional interaction or introducing any occlusions. Moreover, preserving both the proximity information (e.g., geographical position, surrounding areas) due to avoiding occlusion, and the faraway contextual information (e.g., connections to other nodes in the network or to SCADA appliances near or far the controlling nodes), the security operator is able to 
monitor the whole network status from both logical and geographical perspective integrated in the same view. The versatility of this approach allows to weight the importance of the geographical and topological information to the desired degree by choosing the right cut parameters, with the possibility at any time to re-obtain only the geographical view or the logical view filtering unnecessary details.

\subsection{Subway Exits Scenario}

One of the main profitable situations for using SurgeryCuts is the case in which it is needed to insert textual information on a map that refers to several locations close to the cut center, like labeling the exits of a large central subway station. Displaying such data directly on the map may result in visual occlusion with other labels, making hard to read street names or shops. The user is underground at her arrival station and she needs to make a decision on the exit to get the right bus stop or the shop she is looking for. The greedy approach "let's go out and look where I am" can produce disappointing results, exiting very far from the intended location. Supporting this task requires to have each exit icon on the map labeled with a) the exit name, useful to get direction while moving underground, and b) the list of buses stops close to it, information that is very useful when traveling by subway and buses. As an example, Figure 8 shows a SurgeryCut on the Milano Duomo subway station that encompasses 22 exits on a huge square and adjacent streets. The cut bears all needed exit/buses needed information. Conversely, Figure 9, shows the same information over-imposed on the map using a font of the same size of surrounding labels, producing some clutter.

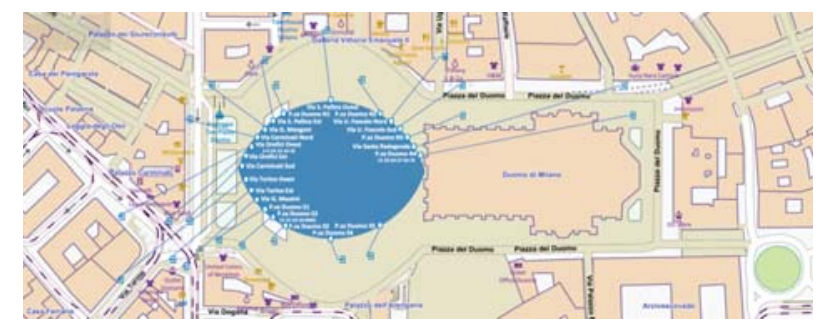

Figure 8: The Milan Duomo subway exits labeled within a cut, together with additional information about adjacent bus stops. The textual information is linked to exits locations through blue lines, making easy to locate shops and other facilities.

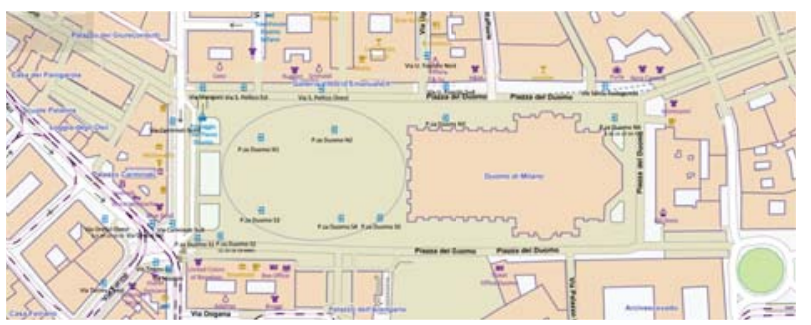

Figure 9: The Milan subway exits labeled with names and list of the buses they are close to (using the same font size of the map labels and minimizing occlusion). The resulting clutter makes hard to read exit names and buses information (see the bottom left part of the image). A dashed ellipse shows the cut location and size; displaying the textual information on that area will hide 5 subway exits.

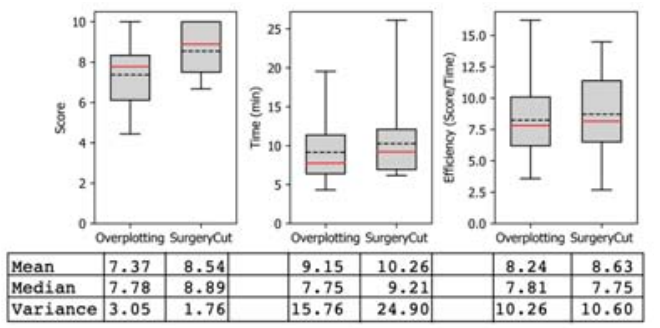

Figure 10: Effectiveness, completion time, and efficiency of the participants. The box-plot on the left shows the distribution of the average scores obtained by counting participants' correct answers and normalizing that value to 10. The box-plot on the middle depicts the distribution of the average time spent to complete the experiment (in minutes). The box-plot on the right illustrates the ratio score/time.

\section{User Study}

We have conducted a controlled experimentvto compare the SurgeryCuts Effectiveness and Efficiency against an occlusion-based labeling technique. We did not compare with other state of the art space warping techniques because they do not provide means for adding new data on the map, nor with Routezoom [SLQW17] and SpaceCut [BJSK16] because they are not general enough, needing the mandatory presence of a map structure, like a road or a river. To the best of the authors' knowledge, the unique technique able to introduce new data on a map without using map features is labelling with occlusion, technique that we have applied minimizing the overlapping with labels used in the study questions. The experiment involved 44 people ( 31 males, 13 females) with no specific skills and used the subway exits scenario described in Section 5.2, providing one interface for the map with a SurgeryCut (see Figure 8) and one in which the 22 exit names have been overplotted on the map, close to the icons of the exits (see Figure 9). To correctly guide the user along the experiment execution steps, i.e., reading questions, interacting with the maps, and reporting responses on a questionnaire, we have used STEIN [ABLS18], an environment that allows for quickly integrating the system under evaluation with the user study questions, tracing her or his activities.

Methodology To compare the two strategies, people were automatically split in two groups: Group 1, performing tasks on the overplotted map, and Group 2, performing the same tasks on the map with the SurgeryCut. Participants have been asked to answer 10 questions (detailed in the additional material) about the 22 exits names, bus lines, and facilities displayed on the map (shops, restaurant, etc.). The first question was associated with the explanation of the environment: participants were asked to inspect the map associated with their group, to get familiar with symbols, and to answer a simple question to assess their understanding. After that, participants answered the remaining 9 questions that have been used for assessing their performances. However, users not correctly answering the first question (or not answering it at all) have been excluded: either they did not understand the explanation or they were answering the questionnaire in a rush or sloppy fashion. That reduced the number of questionnaires to 35 .

Results From traces and answers we have collected three dependent variables: Effectiveness (score), Time, and Efficiency (score/time), whose values are reported as box plots on Figure 10. 
In order to validate the statistical significance of these figures we have performed a between subjects one-way ANOVA that confirmed a significant effect of the independent variable "user interface" on Score, at the $p<0.05$ level, $(\mathrm{F}(1,33)=4.85, \mathrm{p}=0.034)$; concerning Time and Efficiency we concluded that the user interface has not a significant effect on them (at the $p<0.05$ level, $(\mathrm{F}(1,33)=0.54$, $\mathrm{p}=0.47$ and $\mathrm{F}(1,33)=0.18, \mathrm{p}=0.68$, respectively). According to that we can conclude that the Effectiveness of Group 2 interacting with a cut (score mean 8.54, median 8.89) is better than Group 1 dealing with an overplotted map(score mean 7.37, median 7.78); on the other hand, we cannot make any assumptions on differences between Group 1 and Group 2 for what concerns Time and Efficiency. We believe that completion time and, as a consequence, efficiency have been affected by two external factors common to both groups: UNUSUAL ICONS: We received several comments about this issue, which we attribute to differences on between the explicit depiction of subway exits by OpenStreetMap versus a more familiar representation by Google Maps without subway exits.

UNINTENDED TASKS: judging from the comments we discovered that several participants used the map for spotting bus stops (poorly depicted on OpenStreetMap) instead of using the textual information below the exit names. Moreover, we see that the highest score differences come from the two tasks asking a) to list exits connected to the 12 bus line, and $b$ ) to count the subway exits; people using the SurgeryCuts map answered with an efficacy almost three times higher than people using the overplotted map (4.4 against 1.6, and 9.4 against 3.2, respectively), confirming the cluttering issue.

\section{Discussion}

The introduction of cuts to maps opens new perspectives on data visualization in spatial contexts. Yet, introducing cuts and distortion into maps also raises challenges and questions we want to discuss in the following together with further research perspectives.

Implications of Distortion When creating a cut, three factors have to be balanced: The size of the ACA to create in two dimensions, and the extent of the area affected by the cut. In other words, there is a trade-off between generated ACA area and the amount of distortion introduced. The more space created, the more local distortion is introduced. Of course, this can be alleviated by increasing the size of the affected area in total, yet increasing the amount of distorted map space. So far, we expect there to be no optimal solution to this trade-off, and the selection of these parameters depends on use case and type of the desired visualization to be put in a cut.

Another concern for distortion areas is the handling of conflicting cuts in multi-cut scenarios. At the moment, as a straightforward solution, our prototype does not allow the creation of conflicting cuts. Yet, Mizutani et al. [MYT13] provide the water drop technique, emulating surface tension conditions to disperse conflicting distortions at focus+glue+context lenses. As a baseline approach, they also propose a force-directed lens repulsion. Such strategies could be integrated with the way SurgeryCuts are created. In future work, we aim to explore this possibility in more detail.

Cut Shape Perception and Zoom Level We see a benefit of SurgeryCuts in the visual representation of a cut as the lenticular shape and the resulting continuous distribution of the distortion create a regular distortion area. This kind of regular, but not completely circular shape is uncommon for most environments and thus makes it easier for observers to identify distorted areas even without focusing at the actual cut. For the same reason, we expect that with the lenticular shape, mistaking the distortion for real structures can be minimized. When zooming out, our current solution is to consider the ACA equal to other map elements. Hence, a cut will shrink or enlarge in accordance with the selected zoom level. However, for practical applications, other approaches can make sense. We recommend considering the importance of the visualized element in context of the zoom level and the importance of the surrounding map features to determine whether a cut should resize with changing zoom levels. Further, changing the granularity of the information visualized in the cut with changing zoom levels is conceivable as well. A possible perceptual complication can arise when a user seeing a cut intersecting an element, e.g. a street, is led to think that the cut is occluding part of the element. Especially for untrained users, this impression might occur for larger cuts. These perceptual effects are related to the Gestalt Laws of continuation, closure and similarity, which can, in turn, be used to alleviate said effects, which we intend to elaborate on in future work. Promising methods include the introduction of visual cues around the cut shape to imply continuity of the post-cut features. As well, continuing important map features like roads into the edge of the cut and fading them out has potential to generate a sense of continuity.

Cut Determination and Generation We have not focused on how to determine size and bearing of a cut on a given location and our demonstrator lets the user determine these parameters fully on his own. However, the choice especially of the bearing of a cut in a specific location can produce considerable visual differences in the result, as Figure 3 shows. Again, the method of choice is dependent on the use case and should be decided accordingly. We envision different approaches to determine the cut: First, all parameters could be decided automatically by the system on request, for example by a click on a composed node in the Panoptesec scenario. Second, a semi-automatic approach could help a user by suggesting parameters according to a location and cut size chosen by the user. Third, the manual generation of the cut is the most viable option to make use of an analysts context knowledge or specific information needs.

\section{Conclusion}

The paper introduced SurgeryCuts, a novel map alteration technique creating additional space for visualizations on maps without occluding map features. In comparison to related approaches, the additional canvas area is structure-independent and does not shift the viewport of the map. The applied distortion is limited to a single axis and designed to distribute the amount of distortion introduced as evenly as possible to limit the perceptional implications of the cut. We discussed the reasoning for a lenticular cut shape and provided a detailed description for the construction of SurgeryCuts. To measure the impact of our technique, we contribute quantitative metrics. We compared our approach to the two most related techniques and exemplified the application of SurgeryCuts in two practical use cases. A controlled user study provided additional insights on the SurgeryCuts potentialities. Since this publication solely introduces SurgeryCuts as fundamental technique itself, we intend to explore the benefits of automatic and interactive approaches to cut generation as discussed in the previous Section 7 in future works on the topic. Finally, we are looking forward to the application of the contributed metrics to perform comparisons of further techniques. 


\section{References}

[AA13] ANDRIENKO G., ANDRIENKO N.: Visual analytics of movement: An overview of methods, tools and procedures. Information Visualization 12, 1 (2013), 3-24. doi : 10.1177/1473871612457601. 10

[ABLS18] Angelini M., Blasilli G., Lenti S., Santucci G.: Stein: speeding up evaluation activities with a seamless testing environment integrator. In Eurographics Conference on Visualization (EuroVis) Short Papers (2018), The Eurographics Association. doi:10.2312/ eurovisshort.20181083. 9

[AS17] ANGELINI M., SANTUCCI G.: Cyber situational awareness: from geographical alerts to high-level management. Journal of Visualization 20, 3 (2017), 453-459. doi : 10 . 1007/s12650-016-0377-3. 8

[BJSK16] Buchmüller J., JÄCKle D., Stoffel F., Keim D. A.: SpaceCuts: Making Room for Visualizations on Maps. In Eurographics Conference on Visualization (EuroVis) - Short Papers (2016), Bertini E., Elmqvist N., Wischgoll T., (Eds.), The Eurographics Association. 10.2312/eurovisshort.20161163. doi : 10.2312/eurovisshort. 20161163. 2, 3, 7, 9

[BS06] Bertini E., Santucci G.: Visual quality metrics. In Proceedings of the 2006 AVI workshop on BEyond time and errors: novel evaluation methods for information visualization (2006), ACM, pp. 1-5. doi:10.1145/1168149.1168159. 6

[CCF97] Carpendale M. S. T., COWPerthwaite D. J., Fracchia F. D.: Making distortions comprehensible. In Visual Languages, 1997. Proceedings. 1997 IEEE Symposium on (1997), IEEE, pp. 36-45. doi : 10.1109/VL. 1997.626556. 3, 5

[CM01] Carpendale M. S. T., Montagnese C.: A framework for unifying presentation space. In Proceedings of the 14th Annual ACM Symposium on User Interface Software and Technology (New York, NY, USA, 2001), UIST '01, ACM, pp. 61-70. URL: http: //doi . acm.org/ 10.1145/502348.502358, doi:10.1145/502348.502358. 5

[CSC06] Correa C., Silver D., Chen M.: Feature aligned volume manipulation for illustration and visualization. IEEE Transactions on Visualization and Computer Graphics 12, 5 (2006), 1069-1076. doi : 10.1109/TVCG.2006.144. 2

[DMK05] DyKes J., MACEACHREN A. M., KRAAK M.-J.: Exploring geovisualization. Elsevier, 2005. doi : 10.1002/arp.274. 2

[Fur86] Furnas G. W.: Generalized fisheye views. In Proceedings of the SIGCHI Conference on Human Factors in Computing Systems (New York, NY, USA, 1986), CHI '86, ACM, pp. 16-23. URL: http://doi . acm.org/10.1145/22627.22342, doi: 10.1145/22627.22342. 10

[GI07] Gustafson S. G., IRANi P. P.: Comparing visualizations for tracking off-screen moving targets. In CHI'07 Extended Abstracts on Human Factors in Computing Systems (2007), ACM, pp. 2399-2404. doi : 10.1145/1240866.1241014. 2

[Gut02] GUTWIN C.: Improving focus targeting in interactive fisheye views. In Proceedings of the SIGCHI conference on Human factors in computing systems (2002), ACM, pp. 267-274. doi : 10.1145/503376. 503424. 3

[HS11] HAUNERT J.-H., SERING L: Drawing road networks with focus regions. IEEE transactions on visualization and computer graphics 17 , 12 (2011), 2555-2562. doi : 10.1109/TVCG.2011.191. 2

[KY95] KURZION Y., YAGEL R.: Space deformation using ray deflectors. In Rendering Techniques 95. Springer, 1995, pp. 21-30. doi: 10.1007/ 978-3-7091-9430-0_3. 2

[Mac04] MACEACHREN A. M.: How maps work: representation, visualization, and design. Guilford Press, 2004. 1, 2

[MRC91] Mackinlay J. D., Robertson G. G., Card S. K.: The perspective wall: Detail and context smoothly integrated. In Proceedings of the SIGCHI conference on Human factors in computing systems (1991), ACM, pp. 173-176. doi : 10.1145/108844.108870. 10

[MYT13] Mizutani H., Yamamoto D., Takahashi N.: A preventing method for overlapping focuses in a focus+ glue+ context map. International Journal of Knowledge and Web Intelligence 4, 1 (2013), 50-69. doi : 10.1504/IJKWI. 2013.052725. 10
[NC03] Neumann P., Carpendale S.: Taxonomy for Discrete Lenses. Tech. rep., 2003. 2

[Pan16] PANOPTESEC CONSORTIUM: The official website of the panoptesec project. http://www . panoptesec . eu/, 2014-2016. 8

[PPCP12] Pindat C., Pietriga E., Chapuis O., Puech C.: Jellylens: content-aware adaptive lenses. In Proceedings of the 25th annual ACM symposium on User interface software and technology (2012), ACM, pp. 261-270. doi : 10.1145/2380116.2380150. 10

[SA82] SPENCE R., ApPeRley M.: Data base navigation: an office environment for the professional. Behaviour \& Information Technology 1, 1 (1982), 43-54. doi : 10.1080/01449298208914435. 2

[SB94] SARKAR M., Brown M. H.: Graphical fisheye views. Communications of the ACM 37, 12 (1994), 73-83. doi : 10.1145/198366. 198384. 2

[SLQW17] Sun G., LIANG R., QU H., WU Y.: Embedding spatiotemporal information into maps by route-zooming. IEEE transactions on visualization and computer graphics 23, 5 (2017), 1506-1519. doi : 10.1109/TVCG.2016.2535234.2,3, 7,9

[SV10] Speckmann B., VerbeeK K.: Necklace maps. IEEE Transactions on Visualization and Computer Graphics 16, 6 (2010), 881-889. doi : 10.1142/s021819591550003x. 2

[Tak08] TAKAHASHI N.: An elastic map system with cognitive mapbased operations. In International Perspectives on Maps and the Internet. Springer, 2008, pp. 73-87. doi : 10.1007/978-3-540-72029-4_5. 2

[TGK*17] Tominski C., Gladisch S., Kister U., Dachselt R., SCHUMANN H.: Interactive lenses for visualization: An extended survey. In Computer Graphics Forum (2017), vol. 36, Wiley Online Library, pp. 173-200. doi:10.1111/cgf.12871. 2

[YOT09] Yamamoto D., OzEKI S., TAKahashi N.: Focus+ glue+ context: An improved fisheye approach for web map services. In Proceedings of the 17th ACM SIGSPATIAL International Conference on Advances in Geographic Information Systems (2009), ACM, pp. 101-110. doi : $10.1145 / 1653771.1653788 .10$ 